\title{
Sobrecarga de familiares cuidadores de pacientes psiquiátricos: relação com a resiliência
}

Luís Fernando de Abreu Porto

Marina Bandeira

Marcos Santos de Oliveira

\section{RESUMO}

Com a desinstitucionalização psiquiátrica, intensificou-se o papel da família no cuidado cotidiano dos pacientes, resultando em sentimento de sobrecarga. 0 modelo teórico da sobrecarga prediz que ela poderia ser diminuída por fatores moduladores, como a resiliência, definida como a habilidade para enfrentar adversidades, ajustando-se de forma positiva. Este estudo investigou a relação entre o nível de resiliência e o grau de sobrecarga desses cuidadores. Foram entrevistados 76 familiares de pacientes atendidos em três serviços de saúde mental de duas cidades de Minas Gerais. Foram obtidas correlações significativas entre o nível de resiliência e dois domínios da sobrecarga (assistência na vida cotidiana e supervisão dos comportamentos problemáticos), mas não em relação às preocupações com o paciente, ou o escore global de sobrecarga. Foram identificados os principais fatores associados à sobrecarga, características dos pacientes e dos familiares. Conclui-se pela necessidade de intervenções psicoeducativas dos serviços de saúde mental junto a esses cuidadores. 


\section{W'INTERACÃO EM ET. PSICOLOGIA}

encontradas altas taxas de prevalência de transtornos psiquiátricos na população adulta brasileira, variando entre $20 \%$ e $56 \%$.

Os transtornos psiquiátricos possuem, ainda, um elevado ônus, pois são responsáveis por $13,1 \%$ da Carga Global de Doenças. Estsa medida considera a soma dos anos de vida perdidos por morte prematura aos anos vividos com incapacidade, ajustados de acordo com a gravidade do transtorno (WHO, 2008). No Brasil, estima-se que os transtornos psiquiátricos contribuem com 20,3\% da Carga Global de Doenças (WHO, 2011). Considerando-se apenas os anos vividos com incapacidade, esste índice atinge entre 40 a 48\% (Kohn, Mello, \& Mello, 2007). Pessoas com transtornos psiquiátricos possuem taxas maiores de mortalidade quando comparados à população em geral. Além disso, as consequências econômicas dessas perdas são também altas: um estudo recente estimou que o impacto global cumulativo dos transtornos psiquiátricos em termos de perdas econômicas será da ordem de US\$ 16.3 trilhões entre 2011 e 2030 (WHO, 2013).

0 ônus dos transtornos psiquiátricos atinge diretamente as famílias, pois com o processo da reforma psiquiátrica, o tratamento das pessoas com transtornos psiquiátricos graves e persistentes deslocou-se progressivamente dos hospitais para a comunidade. Como consequência, os familiares passaram a se envolver mais com o cuidado cotidiano desses pacientes, desempenhando um papel importante para a sua reabilitação social (Bandeira \& Barroso, 2005; Cardoso, Galera, \& Vieira, 2012; Morgado \& Lima, 1994; OMS, 2001).

O maior envolvimento da família no cuidado dos pacientes resultou em um sentimento de sobrecarga para os familiares cuidadores, o que pode comprometer seu bemestar físico e psíquico, qualidade de vida e impactar o funcionamento da família como um todo. Essa sobrecarga pode ser agravada devido às dificuldades dos serviços de saúde mental em atender satisfatoriamente às múltiplas demandas desses pacientes no processo de reinserção social (Bandeira \& Barroso, 2005; Barroso, 2014; Tessler \& Gamache, 2000; Zauszniewski, Bekhet, \& Suresky, 2010).

0 conceito de sobrecarga refere-se às consequências negativas, para o familiar cuidador, resultantes da presença do transtorno psiquiátrico na família (Bandeira \& Barroso, 2005). De acordo com o modelo teórico elaborado por Maurin e Boyd (1990), alguns fatores são moduladores da sobrecarga. A capacidade de construir uma rede de suporte social, de utilizar estratégias adequadas de enfrentamento e de ter um bom relacionamento com o paciente podem reduzir a sobrecarga. Nesse contexto, Saunders (2003) e Grotberg (2005) apontam que a resiliência também pode ser um fator modulador da sobrecarga. A resiliência pode ser definida como a habilidade de um indivíduo para se recuperar das adversidades, ajustando-se de forma positiva, mesmo em face de situações de estresse e tensão (Lopes \& Martins, 2011). Com base nesse modelo teórico, pode-se prever, portanto, uma relação inversa entre o nível de resiliência dos familiares e seu grau de sobrecarga subjetiva no desempenho do papel de cuidadores.

Para identificar estudos que tenham avaliado a relação entre resiliência e sobrecarga de familiares cuidadores de pacientes psiquiátricos, foi realizada uma busca nos indexadores Scielo, Psyclnfo, MedLine, PubMed e ScienceDirect, utilizando-se os descritores "sobrecarga", "resiliência", "saúde mental", "familiares cuidadores", "pacientes psiquiátricos", e os termos em inglês " 


\section{N"INTERACÃO EM ET PSICOLOGIA}

pacientes psiquiátricos, atendidos pelo Núcleo de Saúde Mental e pelos Centros de Atenção Psicossocial Tipo I e Tipo II, de duas cidades de porte médio de Minas Gerais. Foram incluídos familiares de ambos os sexos, acima de 18 anos, que residiam com os pacientes, eram seus principais cuidadores e cuidavam do paciente há, pelo menos, um ano. Os pacientes deviam ter diagnósticos de transtorno psiquiátrico, nas categorias F20 a F29 (esquizofrenia, transtornos esquizotípicos e transtornos delirantes) ou F30 a F39 (transtornos do humor ou afetivos), segundo a Classificação Internacional de Doenças (CID-10) (OMS, 1998).

Pelo cálculo estatístico realizado para definir o tamanho da amostra, era necessário entrevistar 71 sujeitos para se obter um poder amostral de $90 \%$, segundo a fórmula de Miot (2011). Foram contatados, ao todo, 97 familiares, porém 21 se recusaram a participar, sendo entrevistados 76 familiares, número superior ao valor mínimo estimado de 71 sujeitos.

\section{INSTRUMENTOS DE MEDIDA}

Serão descritos abaixo os instrumentos de medida utilizados para a coleta de dados.

Escala de Resiliência de Connor-Davidson para Brasileiros (RISC-10-Br): Essa escala foi desenvolvida por Connor e Davidson (2003), sendo adaptada e validada para o contexto brasileiro por Lopes e Martins (2011). É composta por 10 itens que avaliam a percepção dos sujeitos quanto à sua capacidade de adaptação às mudanças, superação de obstáculos, capacidade de recuperação após doenças, lesões e outras dificuldades. As alternativas de resposta estão dispostas em uma escala tipo 


\section{N"INTERACÃO EM \\ ET. PSICOLOGIA}

Pearson entre os escores da FBIS-BR e da escala RISC-10-Br, para verificar a relação entre o nível de resiliência e o grau de sobrecarga subjetiva. Foram feitas, também, análises estatísticas univariadas, com $o$ teste $t$ de 
incomodo, a média global obtida foi de 2,71, em uma escala de 1 a 4 , sendo igualmente moderada. $\mathrm{Na}$ análise das dimensões da sobrecarga objetiva, o teste ANOVA de medidas repetidas indicou que a sobrecarga objetiva foi significativamente superior para a subescala $A$, que avalia a frequência da assistência na vida cotidiana $(M=2,59$; $D P=$ 1,09). 0 teste $t$ de Student, para amostras pareadas, indicou que a sobrecarga subjetiva, por sua vez, foi estatisticamente superior para a subescala $B$, referente ao sentimento de incomodo na supervisão dos comportamentos problemáticos $(M=3,18 ; D P=0,83)$. Esses dados indicam que, embora os familiares tivessem uma frequência menor da atividade de lidar com os comportamentos problemáticos dos pacientes, eles se sentiam mais incomodados com esta tarefa. A sobrecarga relativa às preocupações com o paciente teve média de 3,25 ( $D P=0,97)$, mas esse escore não pode ser comparado aos outros, pois suas opções de resposta diferem das demais.

Uma análise mais detalhada por itens específicos identificou níveis elevados de sobrecarga para alguns aspectos dos cuidados. Para a maioria, as atividades de assistência na vida cotidiana que geraram uma sobrecarga objetiva elevada (escores 4 e 5) foram: preparar as refeições para o paciente $(60,6 \%)$, ajudar com a medicação $(50 \%)$, lembrar ou fazer as tarefas de casa para o paciente $(47,4 \%)$ e lembrar ou insistir para que o paciente ocupasse seu tempo com alguma coisa (46\%). Por sua vez, os comportamentos problemáticos que geraram sobrecarga objetiva elevada foram: tentativas de impedir o paciente de ingerir excessivamente líquidos não-alcoólicos e/ou fumar demais $(42,1 \%)$ e exigências excessivas de atenção $(28,9 \%)$.

Para a sobrecarga subjetiva, os resultados da análise por itens mostraram também uma sobrecarga elevada para alguns aspectos do papel de cuidador. Para a maioria, as atividades da assistência na vida cotidiana que mais provocaram sentimento de incomodo (escores 3 e 4) foram: ajudar ou lembrar o paciente sobre sua higiene pessoal (50\%), administrar a tomada de medicamentos (42,8\%), ajudá-los a fazer compras (42,6\%), ajudar nas tarefas de casa $(38,7 \%)$ e insistir para que os pacientes se ocupassem com alguma atividade (34\%). Por sua vez, os comportamentos problemáticos dos pacientes que mais geraram sentimento de incômodo para a maioria dos cuidadores foram: uso de drogas ilícitas (100\%); lidar com os comportamentos noturnos perturbadores (87,5\%); lidar com a ingestão de bebidas alcoólicas em excesso (87,5\%); lidar com comportamentos desconcertantes do paciente $(86,4 \%)$ e com comportamentos auto $(85,7 \%)$ e heteroagressivos $(84,2 \%)$. Em relação às preocupações com o paciente, os itens mais frequentes (escores 4 e 5) foram: sentir preocupação em relação à saúde física do paciente $(63,2 \%)$; com seu futuro $(63,2 \%)$; com sua segurança física $(61,9 \%)$; com suas condições financeiras $(56,6 \%)$ e com a vida social do paciente (50\%).

\section{CORRELAÇÃO ENTRE RESILIÊNCIA E SOBRECARGA}

As análises correlacionais entre os níveis de resiliência e de sobrecarga subjetiva são apresentados na Tabela 1 . Obteve-se uma correlação significativa entre o nível de resiliência e a sobrecarga referente à assistência na vida cotidiana (subescala $A$ ) e à supervisão dos comportamentos problemáticos (subescala $B$ ). Esses resultados indicam que quanto maior era o nível de resiliência dos familiares, menor o grau sentimento de incômodo na prestação de assistência na vida cotidiana e na supervisão dos comportamentos problemáticos dos pacientes. Entretanto, não houve correlação significativa com a subescala $E$, relativa às preocupações com o paciente, nem com o escore global de sobrecarga subjetiva. Portanto, a hipótese do presente estudo foi apenas parcialmente confirmada.

\begin{tabular}{|c|c|c|c|}
\hline Variáveis & $\begin{array}{c}\text { Médias } \\
\text { (sobrecarga) }\end{array}$ & $R$ & $P$ \\
\hline Subescala A Subjetiva & 1,89 & $-0,27$ & $0,03^{*}$ \\
\hline Subescala B Subjetiva & 3,15 & $-0,31$ & $0,02^{*}$ \\
\hline Subescala E Subjetiva & 3,25 & $-0,35$ & 0,77 \\
\hline Sobrecarga Global Subjetiva & 2,71 & $-0,10$ & 0,38 \\
\hline
\end{tabular}




\section{ANÁLISES MULTIVARIADAS: FATORES PREDITORES DO GRAU DE SOBRECARGA}

A Tabela 2 apresenta os resultados das análises de regressão - separadamente, para as variáveis dos familiares e dos pacientes - que foram preditoras do grau de sobrecarga. A variável resiliência foi incluída, mas não permaneceu no modelo final, não afetando, portanto, o grau de sobrecarga quando comparada com outras variáveis dos familiares e dos pacientes.

\begin{tabular}{|c|c|c|c|c|c|c|c|}
\hline Categoria & Fatores & $\begin{array}{l}\text { Beta } \\
\text { (B) }\end{array}$ & $\begin{array}{c}\text { Erro } \\
\text { Padrão }\end{array}$ & $\begin{array}{l}\text { Beta } \\
\text { Pad } \\
(\beta)\end{array}$ & $T$ & $p$ & \\
\hline \multirow{6}{*}{$\begin{array}{l}\text { Variáveis } \\
\text { dos } \\
\text { Familiares }\end{array}$} & Constante & 3,70 & 0,25 & & 14,70 & 0,00 & \multirow{6}{*}{$\begin{array}{l}\mathrm{R}^{2}=0,40 \\
\mathrm{~F}(5,70)= \\
11,16 \\
p= \\
0,000^{*} \\
{[\mathrm{DW}]=2,04}\end{array}$} \\
\hline & $\begin{array}{l}\text { Familiar está satisfeito com o } \\
\text { apoio que tem recebido }\end{array}$ & $-0,90$ & 0,22 & $-0,38$ & $-4,14$ & 0,00 & \\
\hline & Anos em que cuida do paciente & $-0,02$ & 0,01 & $-0,27$ & $-3,01$ & 0,00 & \\
\hline & Convivência com o paciente & $-0,52$ & 0,12 & $-0,40$ & $-4,30$ & 0,00 & \\
\hline & Parentesco com paciente & 0,37 & 0,13 & 0,27 & 2,95 & 0,00 & \\
\hline & Vive com companheiro & 0,24 & 0,12 & 0,18 & 1,92 & 0,06 & \\
\hline \multirow{5}{*}{$\begin{array}{l}\text { Variáveis } \\
\text { dos } \\
\text { Pacientes }\end{array}$} & Constante & 2,94 & 0,29 & & 10,26 & 0,00 & \multirow{5}{*}{$\begin{array}{l}\mathrm{R}^{2}=0,37 \\
\mathrm{~F}(4,71)= \\
12,18 \\
p= \\
0,000^{*} \\
{[\mathrm{DW}]=1,78}\end{array}$} \\
\hline & Diagnóstico (CID) & 0,34 & 0,13 & 0,24 & 2,53 & 0,01 & \\
\hline & $\begin{array}{l}\text { Número de comportamentos } \\
\text { problemáticos }\end{array}$ & 0,06 & 0,02 & 0,41 & 4,17 & 0,00 & \\
\hline & Toma a medicação sozinho & $-0,34$ & 0,13 & $-0,26$ & $-2,66$ & 0,01 & \\
\hline & Idade & $-0,01$ & 0,01 & $-0,23$ & $-2,44$ & 0,02 & \\
\hline
\end{tabular}

Nota: ${ }^{*} p<0,001$

Fatores preditores referentes aos familiares: cinco variáveis referentes aos familiares se mantiveram no modelo de regressão, indicando que a sobrecarga subjetiva era maior diante das seguintes condições, por ordem inversa de importância, com base no valor de Beta padronizado: em primeiro lugar, quando o familiar considerava sua convivência com o paciente difícil ou muito difícil, em seguida quando não estava satisfeito com o apoio recebido do serviço, quando cuidava do paciente há menos tempo, se era pai/mãe do paciente e se vivia com companheiro. Estas cinco variáveis explicaram $40 \%$ da contribuição das características relativas aos familiares sobre o grau de sobrecarga subjetiva.

Fatores preditores referentes aos pacientes: quatro destes fatores se mantiveram no modelo final de regressão, explicando $37 \%$ do grau de sobrecarga. A sobrecarga era maior nas seguintes condições, por ordem inversa de importância, com base no valor de Beta padronizado: em primeiro lugar, quando o paciente apresentava um número maior de comportamentos problemáticos, em seguida quando o paciente não tomava a sua própria medicação, quando o tipo de diagnóstico era relacionado a transtornos do humor (F30 a F39) e quando o paciente era menor idade.

\section{DISCUSSÃO}

Os resultados destacaram o impacto do papel de cuidador dos familiares de pacientes psiquiátricos, principalmente em relação a aspectos específicos dos cuidados prestados. Esses dados corroboram os resultados de estudos nacionais e internacionais que, também, observaram esta sobrecarga (Albuquerque, Cintra, \& Bandeira, 2010; Bandeira \& Barroso, 2005; Barroso et al., 2007; Batista, Bandeira, Gaglia, Oliveira, \& Albuquerque, 2013; Cardoso et al., 2012; Maurin \& Boyd, 1990; Nolasco, Bandeira, Oliveira, \& Vidal, 2014).

Com relação aos escores médios globais moderados de sobrecarga, os resultados se assemelham aos de outros estudos brasileiros, utilizando a mesma escala de medida e mesmo tipo de população alvo. Para a sobrecarga objetiva, os resultados confirmam os obtidos por Albuquerque et al. (2010), Barroso, Bandeira e Nascimento (2007), Batista et al. (2013), Cardoso et al. (2012) e Nolasco et al. (2014). Para a sobrecarga subjetiva, os resultados se assemelham aos dos autores citados acima, além dos estudos de Camilo, Bandeira, Leal e Scalon (2012) e Nolasco et al. (2014). Esse resultado talvez se explique pela utilização da média dos 


\section{W"INTERACÃO EM PSICOLOGIA}

escores, que tende a camuflar os dados específicos, contrariamente à análise de itens específicos.

A sobrecarga objetiva dos familiares foi mais elevada para a assistência aos pacientes na vida cotidiana do que em relação a lidar com seus comportamentos problemáticos. Os familiares tinham que desempenhar mais frequentemente tarefas como preparar refeições, cuidar da medicação e lembrar ou insistir que o paciente ocupasse o tempo com alguma atividade. Resultados semelhantes foram identificados em outros estudos da área (Albuquerque et al., 2010; Barroso et al., 2007; Batista et al., 2013; Camilo et al., 2012; Nolasco et al., 2014). Esses resultados podem ser explicados pela frequência maior dessas atividades na vida cotidiana do que os demais aspectos do papel de cuidador.

Com relação à sobrecarga subjetiva, os resultados indicaram um sentimento maior de incômodo dos familiares ao lidarem com os comportamentos problemáticos dos pacientes do que ao auxiliá-los nas tarefas cotidianas, embora esses comportamentos acontecessem com menor frequência. Os que geraram maior incômodo foram: perturbações noturnas na casa, comportamentos desconcertantes do paciente, comportamentos auto e heteroagressivos, uso constante de cigarros, ingestão de líquidos em excesso, dentre outros. Esses resultados foram observados nos estudos de Albuquerque et al. (2010), Batista et al. (2013), Camilo et al. (2012) e Nolasco et al. (2014). Quanto às preocupações com o paciente, os familiares se preocupavam, principalmente, com a saúde e segurança física do paciente, seu futuro, suas condições financeiras e sua vida social, resultados que foram encontrados por Barroso et al. (2007), Camilo et al. (2012) e Cardoso et al. (2012).

Os resultados da análise de regressão mostraram diversos fatores associados à sobrecarga, embora a resiliência não tenha se mantido no modelo. No que se refere às características dos familiares, observou-se que considerar a convivência com o paciente difícil ou muito difícil foi o principal fator preditivo. Esse resultado corrobora o modelo teórico da sobrecarga de Maurin e Boyd (1990), no qual está previsto que a qualidade do relacionamento com o paciente pode afetar a sobrecarga. Observou-se, também, que cuidar do paciente há menos tempo era um fator preditivo de maior sobrecarga, corroborando os dados do estudo de Hastrup, Berg e Gyrd-Hansen (2011) e do estudo de Mulud e McCarthy (2016). É possível que, com o passar dos anos, o familiar consiga administrar de forma mais eficiente e se habitue às tarefas necessárias ao cuidado. Foi também preditivo de maior sobrecarga os familiares considerarem insatisfatória a ajuda recebida pelos profissionais dos serviços, confirmando dados de Grella e Grusky (1989) e Santos (2010). Quanto ao fator associado referente ao tipo de parentesco, observou-se que familiares cuidadores que eram pais/mães dos pacientes se sentiram mais sobrecarregados do que os de outro tipo de parentesco. Esses dados são corroborados por diversos estudos semelhantes da área (Albuquerque et al., 2010; Barroso, Bandeira, \& Nascimento, 2007; Martínez, Nadal, Beperet, \& Mendióroz, 2000; Zauszniewski, Bekhet, \& Suresky, 2008). O parentesco com o paciente determina o tipo de relacionamento entre eles, o que pode determinar as diferenças no grau de sobrecarga percebida (Jungbauer \& Angermeyer, 2002). Outro fator associado a um maior grau de sobrecarga foi o familiar viver com um companheiro. Esse resultado é contrário ao observado no estudo de GonçalvesPereira et al. (2013), no qual os familiares que não viviam com um companheiro ou não eram casados apresentavam uma sobrecarga mais elevada. Por sua vez, Viana et al. (2013) encontraram que viver com companheiro ou ser casado não prediz a sobrecarga dos familiares cuidadores. Portanto, há necessidade de mais estudos para elucidar esta questão, tendo em vista as diferenças de resultados obtidos pelas pesquisas com relação a essa variável.

No que se refere às características dos pacientes, a análise de regressão identificou também alguns fatores preditores da sobrecarga, embora a resiliência não tenha se mantido no modelo. 0 fator preditor mais importante foi o maior número de comportamentos problemáticos do paciente, em segundo lugar se ele não toma sozinho o medicamento. Na literatura da área, os comportamentos problemáticos são apontados, consistentemente, como um fator diretamente relacionado a um maior grau de sobrecarga subjetiva (Bandeira \& Barroso, 2005; Cardoso et al., 2012; Eloia, Oliveira, Eloia, Lomeo, \& Parente, 2014; Maurin \& Boyd, 1990; Mulud \& McCarthy, 2016; Pratima, Bhatia, \& Jena, 2011), assim como a falta de adesão ao medicamento (Bandeira \& Barroso, 2005; Souza Filho, Sousa, Parente, \& Martins, 2010; Tessler \& Gamache, 2000).

A sobrecarga dos familiares também foi maior quando os pacientes eram mais novos, resultados corroborados por Zauszniewski et al. (2008) e Barroso et al. (2007). Entretanto, Bandeira e Barroso (2005) e Demarco, Nunes, Jardim, Coimbra e Kantorski (2014) encontraram resultados contrários, havendo, portanto, uma contradição na literatura, o que demanda novas pesquisas. Por sua vez, o tipo de diagnóstico também carece de consenso na literatura da área, conforme revisão feita por Bandeira e Barroso (2005). $\mathrm{Na}$ presente pesquisa, transtornos do humor foram relacionados com uma maior sobrecarga, resultado semelhante ao encontrado por Zauszniewski et al. (2008), mas diferente dos encontrados por Gutiérrez-Maldonado e Caqueo-Urízar (2007) e Martens e Addington (2001), que obteve resultado oposto. Por sua vez, Chadda, Singh e 


\section{W'INTERACÃO EM ET. PSICOLOGIA}

Ganguly (2007) não encontraram diferenças na sobrecarga em função destes dois tipos de diagnóstico.

Quanto à relação entre níveis de resiliência e sobrecarga, os resultados da análise correlacional mostraram que um maior nível de resiliência estava significativamente relacionado com menor sobrecarga subjetiva para dois domínios: assistência na vida cotidiana e supervisão dos comportamentos problemáticos dos pacientes. Porém, esta relação não foi observada para o domínio das preocupações com o paciente, nem com o escore global de sobrecarga subjetiva. Esses resultados diferem dos dados de três estudos (Enns et al., 1999; Mulud \& McCarthy, 2016 e Zauszniewski et al., 2009), que encontraram relações significativas entre os escores globais dessas duas variáveis. Como essas pesquisas usaram instrumentos de medida diferentes da sobrecarga, não foi possível comparar os resultados de cada subescala. Além disso, alguns estudos usaram indicadores indiretos da resiliência e não medidas diretas deste construto (Zauszniewski et al., 2009; Richardson, 2002 \& Van Breda, 2001), o que poderia explicar as contradições de resultados.

Nos estudos com outras populações-alvo (Alzheimer, demência e lesão na medula espinhal), os resultados foram divergentes. Em dois deles, foram encontradas correlações significativas entre as duas variáveis (Fernández-Lansac, López, Cáceres, \& Rodríguez-Poyo, 2012; Simpson \& Jones, 2013). O estudo de Córdoba e Poches (2016) não encontrou uma correlação significativa entre as duas variáveis e no estudo de Vagharseyyedin e Molazem (2013), o resultado foi semelhante ao do presente trabalho, pois observou-se correlação da resiliência com a sobrecarga, mas esta relação não se manteve na análise de regressão, quando comparada a outras variáveis.

A hipótese de uma relação entre resiliência e sobrecarga foi confirmada apenas parcialmente. Quando analisada individualmente, constatou-se que um maior nível de resiliência se relacionava com menor grau de sobrecarga em relação a dois domínios: a prestação de cuidados aos pacientes nas tarefas cotidianas e lidar com seus comportamentos problemáticos. Esses resultados mostram que fortalecer a resiliência dos familiares poderia contribuir para seu próprio bem-estar e também daqueles que eles provêm cuidados, nestes dois domínios. Entretanto, quando a resiliência foi analisada em relação ao escore global de sobrecarga, juntamente com outras variáveis, estas últimas foram mais importantes como preditoras da sobrecarga. Este resultado indica que a resiliência pode afetar o grau de sobrecarga, mas que é menos influente quando comparada a outras variáveis.

Os resultados referentes às demais variáveis que afetaram o grau de sobrecarga se referem à dificuldade de relacionamento do cuidador com o paciente, os comportamentos problemáticos dos pacientes, a falta de adesão ao medicamento, o nível de insatisfação dos familiares com os serviços de saúde mental, dentre outras. Esses resultados apontam para a necessidade dos serviços de saúde mental no desenvolvimento de intervenções junto aos familiares, objetivando melhorar sua convivência com os pacientes e oferecer apoio, bem como oferecer orientação sobre como lidar com os comportamentos problemáticos dos pacientes. Essas intervenções devem focar, ainda, nas tarefas específicas de cuidados que foram considerados de elevada sobrecarga pelos familiares, na análise de itens. Os profissionais devem atuar junto aos familiares no cuidado aos pacientes, dando apoio constante no atendimento às suas diversas necessidades no domicílio e na comunidade, atuando nos momentos de crise e na resolução dos problemas de reinserção social. Os resultados sugerem, ainda, que os profissionais devem trabalhar, junto aos pacientes, na adesão ao medicamento e sua importância para o sucesso do tratamento. Atuando em questões sensíveis como estas, que afetam a sobrecarga dos familiares, a satisfação com os profissionais pode aumentar e a sobrecarga diminuir, consequentemente, melhorando a qualidade de vida dos familiares e os cuidados oferecidos por eles aos pacientes. Uma atenção especial deve ser dada a grupos específicos de cuidadores, como aqueles que são pais dos pacientes, que cuidam dos pacientes há menos tempo que cuidam de pacientes menores de idade e os que cuidam de pacientes com diagnóstico de transtornos do humor, os quais apresentaram maior grau de sobrecarga. Esses familiares são mais vulneráveis e necessitam de mais atenção dos profissionais dos serviços, em intervenções focadas para esses grupos.

\section{DECLARAÇÃO DE FINANCIAMENTO}

A pesquisa relatada no manuscrito foi financiada pelos próprios autores.

\section{DECLARAÇÃO DA CONTRIBUIÇÃO DOS AUTORES}

L.F.A.P. realizou a elaboração, coleta e análise de dados da pesquisa; M.B. realizou a orientação da conceitualização, investigação e visualização do artigo; M.S.O. contribuiu com a orientação das análises estatísticas. L.F.A.P. e M.B. fizeram a redação inicial do artigo (rascunho) e são os responsáveis pela redação final (revisão e edição). 
DECLARAÇÃO DE CONFLITOS DE INTERESSE

Os autores declaram que não há conflitos de interesse no manuscrito submetido.

\section{REFERÊNCIAS}

Albuquerque, E., Cintra, A., \& Bandeira, M. (2010). Sobrecarga de familiares de pacientes psiquiátricos: Comparação entre diferentes tipos de cuidadores. 


\section{W'INTERACÃO EM \\ ET. PSICOLOGIA}

Jungbauer, J., \& Angermeyer, M. (2002). Living with a schizophrenic patient: a comparative study of burden as it affects parents and spouses. 
